\title{
Study on an Embedded Monitoring and Control System of Tower Crane
}

\author{
Jianzhi Wang ${ }^{1, a}$, Baojiang Chen ${ }^{2, b}$ and Lin $\mathrm{Xu}^{3, \mathrm{c}}$ \\ ${ }^{1}$ School of Mechanical-electronic and Automobile Engineering \\ Beijing University of Civil Engineering and Architecture \\ Beijing, China \\ ${ }^{2}$ School of Mechanical-electronic and Automobile Engineering \\ Beijing University of Civil Engineering and Architecture \\ Beijing, China \\ ${ }^{3}$ School of Mechanical-electronic and Automobile Engineering \\ Beijing University of Civil Engineering and Architecture \\ Beijing, China

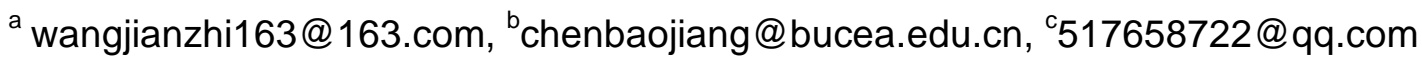

\begin{abstract}
Keywords: tower crane; monitoring and control system; module embedded; CRIO;
Abstract. The monitoring and control system of tower crane is an embedded system which is based on the CRIO module and LabVIEW platform. It can monitor the work condition parameters and control the executing elements of tower crane. The embedded system configures CAN bus driver module and CAN node field detection module, which are reconfigurable and easy to be expanded. This system lays technique foundation for the great promotion of intelligent and informatization of our country's tower crane and other kinds of crane.
\end{abstract}

\section{Introduction}

With the development of high-tech, control technology has been widely used in construction machinery industry. Countries are scrambling to carry out research in the monitoring and control system to enhance the level of automation and efficiency. The real-time embedded monitoring and control system can be used to greatly enhance the automation and intelligence level of the heavy machinery by the way of real-time collecting data, controlling, communicating, and processing video images. The tower crane, short for tower slewing crane, is a common large-scale construction machinery, and it usually be used in non-continuous transport process. The advantage of tower crane is that it can accurately transport the components or materials to any position of the building. However, because of its high center of gravity, large lifting capacity and quick speed, the tower crane is also a kind of machinery with hidden risk factors, so it should be real-time monitored during its operation.

\section{Overall design}

The system takes the programmable automation controller CRIO NI as the core, including the main module, the input module, the output module and the CAN bus driver module.

Programmable automation controller collects circular information of the tower crane by the input module, and displays by the Ethernet on a PC or a touch screen of interactive device after arithmetic processing. Meanwhile, according to the control requirements, the CRIO programmable controller controls actuator machine of the tower crane by the output module, and automates its operation.

The introduction of CAN module brings great flexibility to the system especially for the large-scale construction machinery which has more collection control nodes and far transmission distance. The data collection and actuator control are achieved through the intelligent nodes which are articulated to the bus. 


\section{Module Design}

CPU Module. The paper uses the CRIO-9012 NI as the control core of the embedded system, and it comes from the American National Instrument Company. It uses reconfigurable I/O and FPGA chip and is adapted to reliable independent embedded or distributed application systems. At the same time, it also includes hot-swappable industrial I/O modules and signal condition which can connect the built-in sensor / actuator directly.

Basic Input Module. The basic input module includes analogue input module and digital input module. For the analogue, when the sensor turns the non-electrical signals into the weak electrical signal, the weak signal needs to amplify to improve the resolution and match the ADC voltage range with conditioned voltage range. Thus the analog input module circuit consists of three parts: a voltage follower circuit, polarity switching circuit and scaling circuit. And the three parts above of the circuit are made LM324AN operational amplifier as the core component.

NI9201 from NI Company is chosen as the analogue input module. Its input voltage is $\pm 10 \mathrm{~V}$, and the channel number is 8 , and its characteristics are: 12-bit, 500kS/s aggregate sampling rate, multiplexed election and single ended.

In order to improve anti-jamming capability of the system, the solation design is used for the digital input module. TLP521-1 is chosen as the Opt coupler isolation element and its forward current IF is $70 \mathrm{~mA}$, the forward voltage drop VF is $1.0 \mathrm{~V}-1.3 \mathrm{~V}$.

Basic Output Module. After the data collection, the CRIO programmable controller is used to do a series of arithmetic processing, and then using the drive circuit to drive the solenoid valves and relays, etc. Therefore, the design of the basic output module is to mainly design its drive circuit.

In particular, for the analogue quantity, if the output voltage range of the output module is not in conformity with the required, it need to be buffered, polarity conversed, amplified, isolated and other signal processing before drive the analogue circuit. In this paper, the NI9263 CRIO analogue output module, the output voltage is $-10 \mathrm{~V} \sim+10 \mathrm{~V}$, can be converted using the above method.

For the digital, if the drive capacity of the digital output port is limited, the execute device must be driven through the drive circuit. In this paper, the NI9401 digital output module output capacity is limited, so the same method can be used to match the device above.

CAN Module and Intelligent Node Design. Controller Area Network (CAN) is a controller area network, and it is one of the most widely used field bus around the world. As a kind of large medium construction machinery, the tower crane has a large number of working parameters, and some have a far transmission distance. In order for the tour acquisition parameters, algorithm processing, and real-time output control of these parameters, it's very suitable to use the fieldbus technology. Implanting the CAN module to the measurement and control system of the tower crane can dynamically increase or decrease the intelligent node based on the specific work of the tower crane, and it can bring the system great flexibility and increased its versatility.

The CAN NI-9853 controller module adopted in this paper is a dual port high-speed CAN module with the Philips SJA1000 CAN controller and the Philips TJA1041 CAN transceiver inside. It can achieve hardware synchronization with any CRIO I/O module and transmit / receive all bus loads at the rate of $1 \mathrm{Mb} / \mathrm{s}$.

\section{Analogue I/O and Digital I/O Debug}

Analogue I/O Debug. The voltages of temperature sensor, pressure sensor, torque sensor, angle sensor and angular velocity sensor are collected separately from the channel 0, 1,2,3,4 of the NI 9201, and displayed as the values and the chart (Fig.1) on the debugging interface.

Fig. 2 shows an analogue I/O debugging program which runs on the RT Module. The input and output ports must first be defined on the FPGA, and then the well-defined ports will be displayed on the RT. The general process procedure is as follows: The first step is to open the FPGA, and the second step is to set the sampling rate, and the third is to control the read and write, and the amount defined in the FPGA can all be displayed here. Finally, it is calculated based on the relationship between the 
voltage and collected analogue, and displayed in the form of values and charts. In order to measure the accuracy of the inspection system, comparing the instrument display data(which can be regarded as the true value) and temperature data on the Lab VIEW program interface, the precision of the measurement and control platform test data can be obtained, which can tell whether meet the requirements. Experimental results show that the accuracy of the data meet the requirements.

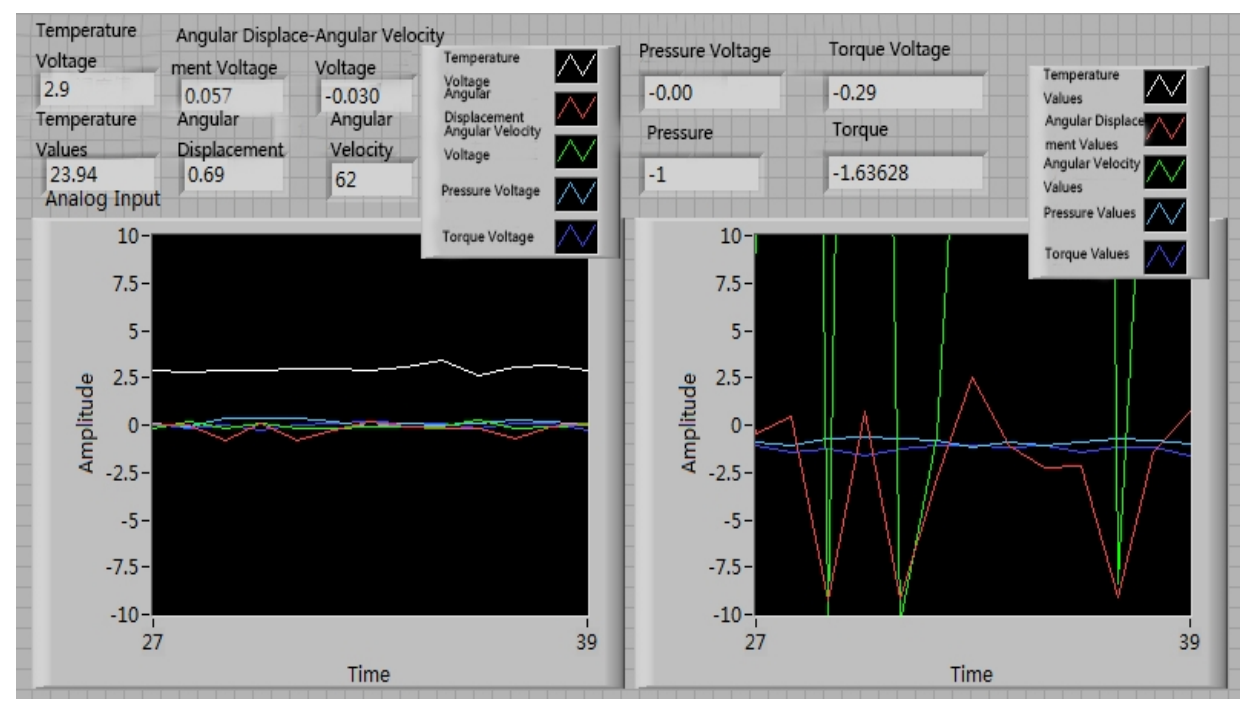

Fig.1 temperature, angle, angular velocity, torque monitoring and debugging interface

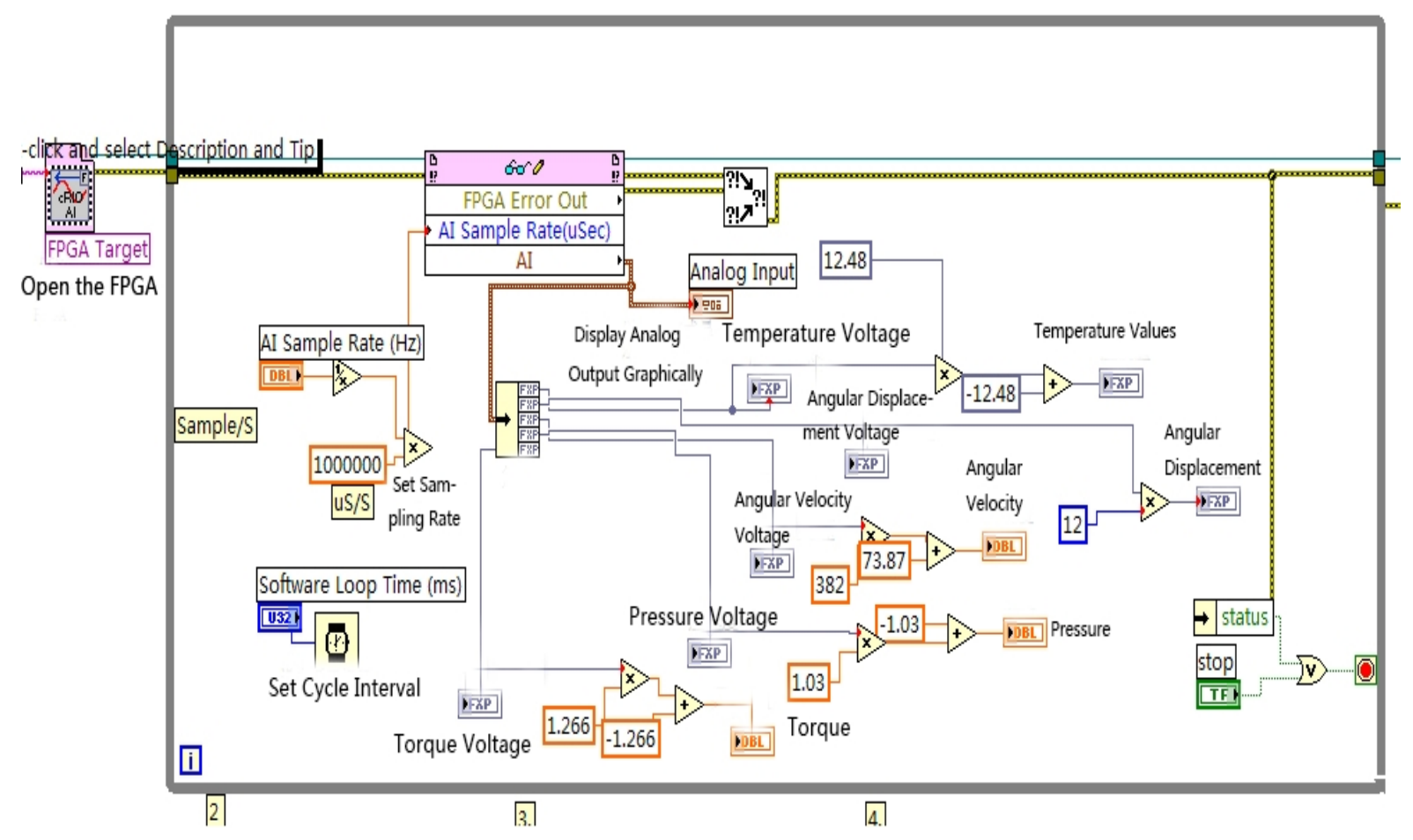

Fig.2 temperature, angle, angular velocity, torque monitoring and debugging program

Digital I/O Debug Proximity switch and photoelectric switch get to $\pm 10 \mathrm{~V}$ voltage signal after the processing of conditioning circuit, and then digital data will be collected from the channel 0,1,2,3 of NI 9401 module. Its debugger interface and block diagram are shown in Fig. 3 . 


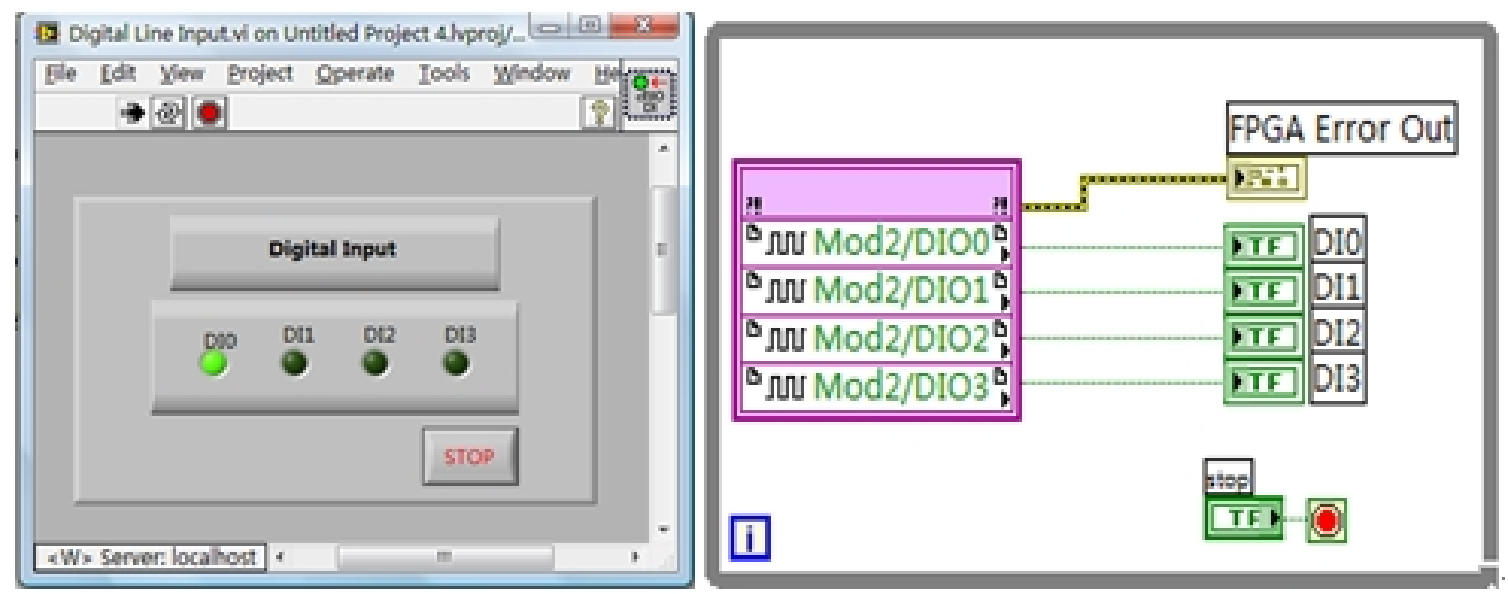

Fig.3 Digital debugging interface and debug the block diagram

In order to test the effectiveness of the program, checking the lights of the proximity switch and photoelectric switch to see whether it is consistent with the Lab VIEW boolean control lights. And by this way, the proximity switch and photoelectric switch also can be debugged. Through the experiments we can draw the following conclusion that NI CRIO can achieve the digital collection and also can meet the system requirem

\section{Conclusions}

Based on the previous research, the paper embodies the research of module integrated embedded systems, and successfully designs and debugs the embedded monitoring and control system of Tower cranes. Conforming to the development direction of the embedded system, the study selects the embedded system with CRIO as the core and Lab VIEW as the develop platform. Compared to conventional PLC, CRIO has a better adaption to the increasing demands for the intelligence of construction machinery. At the same time, CAN fieldbus used for the data collection and real-time control improves the scalability and reconfigurability of this monitoring and control system. If you follow the "checklist" your paper will conform to the requirements of the publisher and facilitate a problem-free publication process.

\section{References}

[1] Baojiang Chen, Jia Mi: On technological current situations, development trends and high-tech demand analysis of large and medium construction machinery [J]. Hoisting and Conveying Machinery, Sept (2008).

[2] Tam V W Y, Fung I W H. Tower crane safety in the construction industry: A Hong Kong study[J]. Safety Science, (2011), 49(2):208-215.

[3] Li L. Improvement to K10 $000 \mathrm{t} \cdot \mathrm{m} / \mathrm{L}-355$ tower crane control system improvement[J]. Hoisting \& Conveying Machinery, (2012).

[4]Jia Mi, Baojiang Chen, Modular integrated embedded monitoring system for heavy construction machi nery[J]. Hoisting and Conveying Machinery(2008)51-53.

[5] Yang W. Development of Intelligent Status Monitoring Instrument for Tower Crane[J]. Electronics \& Automation, (1998). 